\title{
Algebraic cohomology: the early days
}

\author{
Michael Barr \\ barr@barrs.org \\ Department of Mathematics and Statistics, McGill University
}

805 Sherbrooke W, Montreal, QC, H3A 2K6, Canada

\begin{abstract}
This paper will survey the various definitions of homology theories from the first Eilenberg-Mac Lane theories for group cohomology through the Cartan-Eilenberg attempt at a uniform (co-)homology theory in algebra, cotriple cohomology theories and the various acyclic models theorems that tied them all together (as much as was possible).
\end{abstract}

\section{Introduction}

This paper is a mostly historical introduction to the topic of cohomology theories in algebra between 1940 and 1970 when my interests turned elsewhere. This is not to suggest that progress stopped that year, but that I did not keep up with things like crystalline cohomology, cyclic cohomology, etc., and therefore will have nothing to say about them. Much of what I report, I was directly involved in, but anything earlier than about 1962 is based either on the written record or on hearing such people as Samuel Eilenberg and Saunders Mac Lane reminisce about it. Therefore I report it as true to the best of my belief and knowledge.

I have also omitted any mention of sheaf cohomology. I had nothing to do with it and did not know the people most associated with it - Grothendieck, Godement and others. This was much more highly associated with developments in category theory which I knew little about until after the time frame I am dealing with here. The first development here was Mac Lane's paper [1950] which was the first paper to discover universal mapping properties and also attempt to define what we now call abelian categories, later given their full definition in [Buchsbaum, 1956] and [Grothendieck, 1957].

1.1 Acknowledgment. I would like to thank the referee who took an inexcusably careless draft and read it with care and many - far too many than should have been necessary - valuable suggestions for improvements. Any remaining errors and obscurities are, of course, mine.

2000 Mathematics Subject Classification. 18-03, 18G10.

Key words and phrases. Homology and cohomology, triples, acyclic models.

This research has been supported by the NSERC of Canada. 


\section{Eilenberg-Mac Lane cohomology of groups}

2.1 The background. Eilenberg escaped from Poland in 1939 and spent the academic year 1939-40 at the University of Michigan. Mac Lane, then a junior fellow at Harvard, was invited to speak at Michigan. He was attempting to compute what I will call the Baer group of a group $\pi$ with coefficients in a $\pi$-module $A$. This group, which I will denote $B(\pi, A)$, can be described as follows. Consider an exact sequence

$$
0 \longrightarrow A \longrightarrow \Pi \longrightarrow \pi \longrightarrow 1
$$

(Of course, that terminology did not exist in those days; Mac Lane - and Baerwould have said that $A$ was a commutative normal subgroup of $\Pi$ and $\pi=\Pi / A$.) Since $A$ is normal subgroup of $\Pi$, it is a $\Pi$-module by conjugation. Since $A$ is abelian, the action of $A$ on itself is trivial, so that the $\Pi$-action induces a $\pi$-action on $A$, which may, but need not be the action we began with. Then $B(\pi, A)$ is the class of all such exact sequences that induce the $\pi$-action we started with. Say that the sequence above is equivalent to

$$
0 \longrightarrow A \longrightarrow \Pi^{\prime} \longrightarrow \pi \longrightarrow 1
$$

if there is a homomorphism (necessarily an isomorphism) $f: \Pi \longrightarrow \Pi^{\prime}$ such that

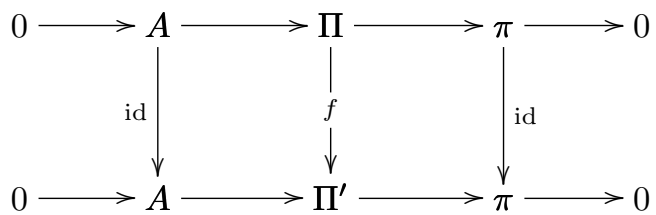

commutes. Then the equivalence classes of such sequences are a set. Moreover there is a way of adding two such equivalence classes that makes $B(\pi, A)$ into an abelian group. If $0 \longrightarrow A \longrightarrow \Pi \longrightarrow \pi \longrightarrow 0$ and $0 \longrightarrow A \longrightarrow \Pi^{\prime} \longrightarrow \pi$ $\longrightarrow 0$ are two such sequences, we first form the pullback $P=\Pi \times_{\pi} \Pi^{\prime}$, let $S$ be the subgroup of all elements of $P$ of the form $(a,-a)$ for $a \in A$. It is immediate that $S$ is a normal subgroup of $P$ and then we can let $\Pi^{\prime \prime}=P / S$. It is easy to see that the two maps $A \longrightarrow \Pi \longrightarrow P$ and $A \longrightarrow \Pi^{\prime} \longrightarrow P$ are rendered equal by the projection $P \longrightarrow P / S$ so there is a canonical map $A \longrightarrow \Pi^{\prime \prime}$. Also the map $P$ $\longrightarrow \pi$ vanishes on $S$ and hence induces $\Pi^{\prime \prime} \longrightarrow \pi$. A simple computation shows that $0 \longrightarrow A \longrightarrow \Pi^{\prime \prime} \longrightarrow \pi \longrightarrow 0$ is exact. That defines the sum of the two sequences. The negative of the sequence $0 \longrightarrow A \longrightarrow \Pi \longrightarrow \pi \longrightarrow 0$ is simply the sequence in which the inclusion of $A \longrightarrow \Pi$ is negated. Since not every element has order 2 this also provides an example showing that equivalence of sequences is not simply isomorphism of the middle term.

Note that in a sequence the kernel $A$ is not merely an abelian group, but also a $\pi$-module. This is because $\Pi$ acts on the normal subgroup $A$ by conjugation and $A$, being abelian, acts trivially on itself so that action extends to an action of $\pi$. The Baer group is the set of equivalence classes of extensions that induce the given module structure on $A$ as a $\pi$-module.

Mac Lane's talk included the computations he was carrying out to compute the Baer group. It would have been along these lines: an extension determines and is determined by a function $\pi \times \pi \longrightarrow A$ satisfying a certain condition and two such functions determined equivalent extensions if there was a function $\pi \longrightarrow A$ that generated the difference of the two original functions. Details will be given later. 
Apparently during Mac Lane's talk, Eilenberg noted that some of the calculations that Mac Lane was carrying out were the same as those he was doing in connection with certain cohomology groups. As they remarked in one of their first papers [Eilenberg and Mac Lane, 1942b], "This paper originated from an accidental observation that the groups obtained by Steenrod [1940] were identical with some groups that occur in the purely algebraic theory of extension of groups." The computations by Steenrod refer to some first cohomology classes (they are described as homology classes of infinite cycles, but I assume that they were really based on duals of chain groups since the dual of an infinite sum is an infinite product, whose elements can look like infinite sums of chains, especially if you do not have a clear idea of contravariant functors) of the complements of solenoids embedded in a 3-sphere. This is likely the second cohomology group of the solenoid itself. See [Eilenberg and Mac Lane, 1941] for more on infinite cycles.

Later in the same paper, in a footnote, they remark, "Group extensions are discussed by Baer [1934], Hall [1938], Turing [1938], Zassenhaus [1937] and elsewhere. Much of the discussion in the literature treats the case in which $G$ but not $H$ is assumed to be abelian and in which $G$ is not necessarily in the centre of $H$." What the latter sentence means is that they were looking at extensions of the form

$$
0 \longrightarrow G \longrightarrow H \longrightarrow H / G \longrightarrow 0
$$

in which $G$ and $H$ are abelian, while the literature was mainly discussing the case that $G$ was an abelian normal subgroup of $H$ and the conjugation action of $H$ (and therefore $H / G)$ on $G$ was non-trivial.

When I gave my talk, I said (and believed, relying on what I thought Eilenberg had said to me 30 years ago, from what he remembered from 30 years before that) that what Eilenberg was working on was calculating the cohomology groups of $K(\pi, 1)$, a space that had fundamental group $\pi$ and no other homotopy. This was described as the main motivation in the later joint work [1947a, b]. But this goes back only to a paper of Hopf's from 1942 in which he showed that if $X$ is a suitable space, then the fundamental group $\pi_{1}(X)$ determines the cokernel of the map from $\pi_{2}(X) \longrightarrow H_{2}(X)$. Thus in the particular case of a $K(\pi, 1)$, whose $\pi_{2}$ is 0 , Hopf's result says that $\pi_{1}(X)$ determines $H_{2}(X)$ (as well, of course, as $H_{1}(X)$, which is the commutator quotient of $\left.\pi_{1}\right)$. It is not clear quite when it was realized that $\pi$ determines all the homology and cohomology groups of a $K(\pi, 1)$, but it must have been before 1947. That paper came after Hochschild's cohomology theory for commutative algebras, which had, it would appear, no connection with topology. I am indebted to Johannes Hübschmann for pointing out some of the pre-history that I was previously unaware of.

2.2 The Eilenberg-Mac Lane groups. Here is a brief description of the Eilenberg-Mac Lane theory. For a non-negative integer $n$, an $n$-cochain on $\pi$ with coefficients in $A$ is a function $f: \pi^{n} \longrightarrow A$. When $n=0$, this is simply an element of $A$. Denote the set of such functions by $C^{n}(\pi, A)$. Define a function $\delta_{n}: C^{n}(\pi, A)$ $\longrightarrow C^{n+1}(\pi, A)$ by

$$
\begin{aligned}
\left(\delta_{n} f\right) & \left(x_{1}, \ldots, x_{n+1}\right)=x_{1} f\left(x_{2}, \ldots, x_{n+1}\right) \\
& +\sum_{i=1}^{n}(-1)^{i} f\left(x_{1}, \ldots, x_{i} x_{i+1}, \ldots, x_{n+1}\right)+(-1)^{n+1} f\left(x_{1}, \ldots, x_{n}\right)
\end{aligned}
$$


The first term uses the action (which could be the trivial, or identity, action) of $\pi$ on $A$. It is a simple exercise to show that $\delta_{n} \circ \delta_{n-1}=0$ so that $\operatorname{im}\left(\delta_{n-1}\right) \subseteq \operatorname{ker}\left(\delta_{n}\right)$ so that we can define

$$
H^{n}(\pi, A)=\operatorname{ker}\left(\delta_{n}\right) / \operatorname{im}\left(\delta_{n-1}\right)
$$

When $n=0$, the cohomology is simply the kernel of $\delta_{0}$. The elements of $\operatorname{ker}\left(\delta_{n}\right)$ are called $n$-cocycles and those of $\operatorname{im}\left(\delta_{n-1}\right)$ are called $n$-coboundaries. Cochains that differ by a coboundary are said to be cohomologous.

2.3 Interpretation of $H^{2}(\pi, A)$. We will see how the group $H^{2}(\pi, A)$ is the same as $B(\pi, A)$. Given an extension

$$
0 \longrightarrow A \stackrel{j}{\longrightarrow} \Pi \stackrel{p}{\longrightarrow} \pi \longrightarrow 1
$$

let $s$ be a set-theoretic section of $p$. This means that $s$ is a function, not necessarily a homomorphism, $\pi \longrightarrow \Pi$ such that $p \circ s=$ id. We can and will suppose that $s(1)=1$. If we write $j(a)=(a, 1)$ for $a \in A, s(x)=(0, x)$ for $x \in \pi$, and $(a, x)$ for $(a, 1)(0, x)$ then the fact that every element of $\Pi$ can be written in the form $j(a) s(x)$ for $a \in A$ and $x \in \Pi$ means that we can identify the underlying set of $\Pi$ with $A \times \pi$. Since $j$ is a homomorphism, we see that $(a, 0)(b, 0)=(a+b, 0)$. Although $s$ is not a homomorphism, $p$ is and that forces the second coordinate of $(0, x)(0, y)$ to be $x y$ so that we can write $(0, x)(0, y)=(f(x, y), x y)$ with $f \in C^{2}(\pi, A)$. Note that $(0, x)(a, 1)=(x a, 1)(0, x)$ since the action of $\pi$ on $A$ is just conjugation. It follows that

$$
\begin{aligned}
(a, x)(b, y) & =(a, 1)(0, x)(b, 1)(0, y)=(a, 1)(x b, 1)(0, x)(0, y) \\
& =(a+x b, 1)(f(x, y), x y)=(a+x b, 1)(f(x, y), 1)(0, x y) \\
& =(a+x b+f(x, y), 1)(0, x y)=(a+x b+f(x, y), x y)
\end{aligned}
$$

Next I claim that the associative law of group multiplication forces $f$ to be a 2 cocyle. In fact, we have for $a, b, c \in A$ and $x, y, z \in \pi$,

$$
\begin{aligned}
((a, x)(b, y))(c, z) & =(a+x b+f(x, y), x y)(c, z) \\
& =(a+x b+f(x, y)+x y c+f(x y, z), x y z)
\end{aligned}
$$

while

$$
\begin{aligned}
(a, x)((b, y)(c, z)) & =(a, x)(b+y c+f(y, z), y z) \\
& =(a+x b+x y c+x f(y, z)+f(x, y z), x y z)
\end{aligned}
$$

and comparing them we see that

$$
f(x, y)+f(x y, z)=x f(y, z)+f(x, y z)
$$

which is equivalent to $\delta_{2} f=0$.

The same computation shows that if we begin with a 2-cocycle $f$ and define multiplication on $A \times \pi$ by the formula

$$
(a, x)(b, y)=(a+x b+f(x, y), x y)
$$

we have an associative multiplication. To see it is a group first suppose that $f(x, 1)=0$ for all $x \in \pi$. It is then an easy computation to show that $(0,1)$ is a right identity and then that $\left(-x^{-1} a-x^{-1} f\left(x, x^{-1}\right), x^{-1}\right)$ is a right inverse for 
$(a, x)$. We will show below that the group extensions constructed using cohomologous cocycles are equivalent. We use that fact here by showing that any cocycle is cohomologous to one for which $f(x, 1)=0$. From

$$
\delta f(x, 1,1)=x f(1,1)-f(x, 1)-f(x, 1)+f(x, 1)=0
$$

we see that $f(x, 1)=x f(1,1)$. Then for $g(x)=f(x, 1)$,

$$
(f-\delta g)(x, 1)=f(x, 1)-x g(1)+g(x)-g(x)=f(x, 1)-x f(1,1)=0
$$

Finally, we claim that equivalent extensions correspond to cohomologous cocycles. For suppose that $f$ and $f^{\prime}$ are 2-cocycles and $g$ is a 1-cochain such that $f-f^{\prime}=\delta g$. Suppose we denote the two possible multiplications by $*$ and $*^{\prime}$ so that

$$
\begin{aligned}
(a, x) *(b, y) & =(a+x b+f(x, y), x y) \\
(a, x) *^{\prime}(b, y) & =\left(a+x b+f^{\prime}(x, y), x y\right)
\end{aligned}
$$

Define $\alpha: A \times \pi \longrightarrow A \times \pi$ by $\alpha(a, x)=(a+g(x), x)$. Then

$$
\begin{aligned}
\alpha((a, x) *(b, y)) & =\alpha(a+x b+f(x, y), x y) \\
& =(a+x b+f(x, y)+g(x y), x y) \\
& =\left(a+x b+f^{\prime}(x, y)+x g(y)+g(x), x y\right) \\
& =(a+g(x), x) *^{\prime}(b+g(y), y) \\
& =\alpha(a, x) *^{\prime} \alpha(b, y)
\end{aligned}
$$

which shows that $\alpha$ is a homomorphism, while it is obviously invertible. Clearly the isomorphism commutes with the inclusion of $A$ and the projection on $\pi$.

Conversely, suppose $f$ and $f^{\prime}$ are cocycles that give equivalent extensions. Again, we will denote the two multiplications by $*$ and $*^{\prime}$. Since we are trying to show that $f$ and $f^{\prime}$ are cohomologous, we can replace them by cohomologous cocycles that satisfy $f(x, 1)=f^{\prime}(x, 1)=0$ for all $x \in \pi$. The fact that the extensions are actually equivalent (not merely isomorphic) implies that the isomorphism $\alpha$ has the property that elements of $A$ are fixed and that the second coordinate of $\alpha(a, x)$ is $x$. In particular, we can write $\alpha(0, x)=(g(x), x)$. Then

$$
\begin{aligned}
\alpha(a, x) & =\alpha\left((0, x) *\left(x^{-1} a, 1\right)\right)=\alpha(0, x) *^{\prime} \alpha\left(x^{-1} a, 1\right) \\
& =(g(x), x) *^{\prime}\left(x^{-1} a, 1\right)=(a+g(x), x)
\end{aligned}
$$

Then from

$$
\begin{aligned}
(a+x b+f(x, y)+g(x y), x y) & =\alpha(a+x b+f(x, y), x y) \\
& =\alpha((a, x) *(b, y)) \\
& =\alpha(a, x) *^{\prime} \alpha(b, y) \\
& =(a+g(x), x) *^{\prime}(b+g(y), y) \\
& =\left(a+g(x)+x b+x g(y)+f^{\prime}(x y), x y\right)
\end{aligned}
$$

we conclude that $f-f^{\prime}=\delta g$. 
2.4 Interpretations in other low dimensions. The definition of $H^{0}(\pi, A)$ as the kernel of $\delta_{0}$ makes it obvious that it is simply

$$
\{a \in A \mid x a=a \text { for all } x \in \pi\}
$$

otherwise known as the group of fixed elements of $A$ and denoted $A^{\pi}$.

The kernel of $\delta_{1}$ consists of those maps $d: \pi \longrightarrow A$ such that $d(x y)=x d(y)+$ $d(x)$. In the case that $\pi$ acts as the identity on $A$, this is exactly a homomorphism of $\pi$ to the abelian group $A$. For this reason, such a function is sometimes called a crossed homomorphism. We prefer to call it a derivation for compatibility with other examples. The image of $\delta_{0}$ consists of those derivations of the form $d(x)=$ $x a-a$ for some element $a \in A$. These are called the inner derivations and $H^{1}(\pi, A)$ is simply the quotient group of derivations modulo inner derivations.

There is also an interpretation of $H^{3}$ that gave information (more limited than $H^{2}$ ) for extensions with non-abelian kernels. We give no proofs here, but content ourselves with a brief description.

Suppose that

$$
1 \longrightarrow G \longrightarrow \Pi \longrightarrow \pi \longrightarrow 1
$$

is an exact sequence of groups. This means that $G$ is a normal subgroup of $\Pi$ and the quotient is $\pi$. Since $G$ is normal, $\Pi$ acts on $G$ by conjugation. This gives a map $\Pi \longrightarrow \operatorname{Aut}(G)$ the group of automorphisms of $G$. Unless $G$ is commutative, this does not vanish on $G$ and hence does not give a natural map $\pi \longrightarrow \operatorname{Aut}(G)$. However, if $\operatorname{In}(G)$ denotes the (normal) subgroup of $\operatorname{Aut}(G)$ consisting of the inner automorphisms, then the composite map $\Pi \longrightarrow \operatorname{Aut}(G) \longrightarrow \operatorname{Aut}(G) / \operatorname{In}(G)$ does vanish on $G$ and hence induces a natural map $\theta: \pi \longrightarrow \operatorname{Aut}(G) / \operatorname{In}(G)$. We will say that $\theta$ is induced by $(*)$.

Now let $\mathscr{Z}(G)$ denote the centre of $G$. One may check that the centre of a normal subgroup is also a normal subgroup so that $\mathscr{Z}(G)$ is also a $\Pi$-module, but one that $G$ acts trivially on, so that $\mathscr{Z}(G)$ is a $\pi$-module. Now it turns out that any homomorphism $\theta: \pi \longrightarrow \operatorname{Aut}(G) / \operatorname{In}(G)$ induces, after a number of choices, a cocycle in $C^{3}(\pi, \mathscr{Z}(G))$ whose cohomology class we denote $[\theta]$.

A homomorphism $\theta: \pi \longrightarrow \operatorname{Aut}(G) / \operatorname{In}(G)$ induces a $\pi$-module structure on $\mathscr{Z}(G)$. Simply choose, for each $x \in \pi$, an element $\bar{\theta}(x) \in \operatorname{Aut}(G)$ whose class mod $\operatorname{In}(G)$ is $\theta(x)$ and define $x z=\bar{\theta}(x)(z)$ for $z \in \mathscr{Z}(G)$. This is well-defined since inner automorphisms are trivial on the centre.

The main result is contained in the following.

Theorem 1 Suppose $G$ is a group whose centre $\mathscr{Z}(G)$ is a $\pi$-module and that $\theta: \pi \longrightarrow \operatorname{Aut}(G) / \operatorname{In}(G)$ is a homomorphism that induces the given action of $\pi$ on $\mathscr{Z}(G)$. Then

1. the cohomology class $[\theta]$ in $H^{3}(\pi, \mathscr{Z}(G))$ does not depend on the arbitrary choices made;

2. the cohomology class $[\theta]=0$ if and only if $\theta$ comes from an extension of the form $(*)$;

3. the equivalence class of extensions $(*)$ that give rise to a given cohomology class $[\theta]$ are in 1-1 correspondence with the elements of $H^{2}(\pi, \mathscr{Z}(G))$; and

4. given a $\pi$-module $A$, every element of $H^{3}(\pi, A)$ has the form $[\theta]$ for some group $G$ and some homomorphism $\theta: \pi \longrightarrow \operatorname{Aut}(G) / \operatorname{In}(G)$ such that $A \cong$ $\mathscr{Z}(G)$ as $\pi$-modules, the latter with the $\pi$-action induced by $\theta$. 
The 1-1 correspondence in the third point above is actually mediated by a principal homogeneous action of $H^{2}(\pi, \mathscr{Z}(G))$ on extensions $(*)$ ([Barr, 1969]). The class $[\theta]$ is called the obstruction of $[\theta]$ (to arising from an extension) and the last clause says that every element of $H^{3}(\pi, A)$ is the obstruction to some homomorphism's coming from an extension.

The most striking application of that theory was that if $\mathscr{Z}(G)=1$, then the equivalence classes of extensions

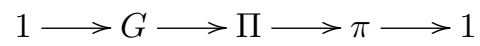

is in 1-1 correspondence with the homomorphisms

$$
\theta: \pi \longrightarrow \operatorname{Aut}(G) / G
$$

\section{Hochschild cohomology of associative algebras}

Gerhard Hochschild defined a cohomology theory for associative algebras in [1945, 1946]. Formally, his definitions look almost identical to those of Eilenberg and Mac Lane.

The setting of this theory is that of an associative algebra $\Lambda$ over a (commutative) field $K$. It was later observed that the definitions can be given when $K$ is any commutative ring, but the resultant extension theory is limited to those extensions that split as $K$-modules. Let $A$ be a two-sided $\Lambda$-module. Define $C^{n}(\Lambda, A)$ to be the set of all $n$-linear functions $\Lambda^{n} \longrightarrow A$. Define $\delta_{n}: C^{n}(\Lambda, A) \longrightarrow C^{n+1}(\Lambda, A)$ by the formula

$$
\begin{array}{rl}
\left(\delta_{n} f\right)\left(x_{1}, \ldots, x_{n+1}\right)=x_{1} & f\left(x_{2}, \ldots, x_{n+1}\right) \\
& +\sum_{i=1}^{n}(-1)^{i} f\left(x_{1}, \ldots, x_{i} x_{i+1}, \ldots, x_{n+1}\right) \\
& +(-1)^{n+1} f\left(x_{1}, \ldots, x_{n}\right) x_{n+1}
\end{array}
$$

This differs from the formula for group cohomology only in that the last term is multiplied on the right by $x_{n+1}$. One can make the formulas formally the same by making any left $\pi$-module into a two-sided $\pi$-module with the identity action on the right. Alternatively, you can use two-sided modules, using the same coboundary formula as for the Hochschild cohomology. This does not really give a different theory, since you can make a two-sided $\pi$-module into a left $\pi$-module by the formula $x * a=x^{-1}$ without changing the cohomology.

Much the same interpretation of the low dimensional cohomology holds for the Hochschild cohomology as for groups. Derivations are defined slightly differently: $d(x y)=x d(y)+d(x) y$, that is the Leibniz formula for differentials. Commutative normal subgroups are replaced by ideals of square 0 .

The limitation to extensions that split as modules was avoided by having two kinds of cocycles (in degree $2 ; n$ in degree $n$ ), one to express the failure of additive splitting and one for the multiplication. This was first done by Mac Lane [1958] and then generalized to algebras in [Shukla 1961].

\section{Chevalley-Eilenberg cohomology of Lie algebras}

The third cohomology theory that was created during the half decade after the war was the Chevalley-Eilenberg cohomology of Lie algebras [1948]. The formulas 
are a little different, although the conclusions are much the same. If $\mathfrak{g}$ is a Lie algebra over the field $K$, then a $\mathfrak{g}$-module $A$ is abelian group with an action of $\mathfrak{g}$ that satisfies $[x, y] a=x(y a)-y(x a)$. It can be considered a two-sided module by defining $a x=-x a$, a fact we will make use of when discussing the CartanEilenberg cohomology. A derivation $d: \mathfrak{g} \longrightarrow A$ satisfies $d[x y]=x d(y)-y d(x)$. An $n$-cochain is still an $n$-linear map $f: \mathfrak{g}^{n} \longrightarrow M$, but it is required to alternate. That is $f\left(x_{1}, \ldots, x_{n}\right)=0$ as soon as two arguments are equal. The coboundary formula is also quite different. In fact the coboundary formulas used for groups and associative algebras would not map alternating functions to alternating functions. The definition found by Chevalley and Eilenberg is given by the following formula, in which the hat, ${ }^{\wedge}$, is used to denote omitted arguments.

$$
\begin{aligned}
\delta f\left(x_{0}, \ldots, x_{n}\right)= & \sum_{i=1}^{n}(-1)^{i} x_{i} f\left(x_{0}, \ldots, \hat{x}_{i}, \ldots, x_{n}\right) \\
& +\sum_{0 \leq i<j \leq n}(-1)^{i+j} f\left(\left[x_{i}, x_{j}\right], x_{0}, \ldots, \hat{x}_{i}, \ldots, \hat{x}_{j}, \ldots, x_{n}\right)
\end{aligned}
$$

The formula arose naturally as the infinitesimal version of the de Rham coboundary formula on Lie groups.

The same kinds of interpretations in low dimensions hold as in the other two cases.

4.1 Comments on these definitions. Two of these three definitions arose from topology in which homology and cohomology were, by the 1940s, coming to be well understood. The cohomology of $\pi$ is the cohomology of the space $K(\pi, 1)$ and the cohomology of a real or complex Lie algebra is that of the corresponding Lie group. As far as I know, there was no topological motivation behind Hochschild's theory. Of course, the interpretations in dimensions $\leq 3$ presumably gave some confidence that the basic theory was correct. Nonetheless, the definitions, viewed as purely algebraic formulas, were inexplicable and ad hoc. Among the questions one might raise was the obvious, "What is a module?" To this question, at least, we will give Beck's surprising and entirely convincing answer.

\section{Cartan-Eilenberg cohomology}

When, as a graduate student in 1959, I took a course from David Harrison on homological algebra, the definitions of cohomology of groups and associative algebras given above were the definitions I learned. (If Lie algebras were mentioned, I do not recall it.) The course was mainly concerned with Ext, Tor and the like, leading to a proof of the Auslander-Buchsbaum theorem. I imagine the book of Cartan-Eilenberg [1956] was mentioned, but I do not think I ever looked at it. The purchase date inscribed in own copy is April, 1962, just after I had finished typing my thesis. My thesis was on commutative algebra cohomology, which I will discuss later, and the methods of that book do not work in that case for reasons I will explain.

When I got to Columbia as a new instructor, the Cartan-Eilenberg book was the bible of homological algebra and I gradually learned its methods. Basically, they had found a uniform method for defining cohomology theories that included the three theories I have described. Only later did it become apparent their methods applied only to the three cohomology theories it was based on and not on any other. 
The way that Cartan and Eilenberg had proceeded was based on the observation that in all three cases, for each object $X$ of the category of interest, there was an enveloping algebra $X^{e}$ that had the property that the coefficient modules for the cohomology were exactly the $X^{e}$-modules. And in all three cases, there was some module - call it $X_{J}$ - for which the cohomology groups could be described as $\operatorname{Ext}_{X^{e}}\left(X_{J}, M\right)$.

5.1 Comparison of Cartan-Eilenberg with earlier theories. Here is how you see that the Cartan-Eilenberg (CE) cohomology of a group is the same as the Eilenberg-Mac Lane (EM) cohomology. To compute the EM cohomology of a group $\pi$ with coefficients in a $\pi$-module $A$, you let $C^{n}(\pi, A)=\operatorname{Hom}_{\text {Set }}\left(\pi^{n}, A\right)$. By using various adjunctions, we see that

$$
\begin{aligned}
C^{n}(\pi, A) & =\operatorname{Hom}_{\mathrm{Set}}\left(\pi^{n}, A\right) \cong \operatorname{Hom}_{\mathrm{Ab}}\left(\mathbf{Z}\left(\pi^{n}\right), A\right) \\
& \cong \operatorname{Hom}_{\mathrm{Ab}}\left(\mathbf{Z}(\pi)^{\otimes n}, A\right) \cong \operatorname{Hom}_{\mathbf{Z}(\pi)}\left(\mathbf{Z}(\pi)^{\otimes(n+1)}, A\right)
\end{aligned}
$$

Here $M^{\otimes n}$ stands for the $n$th tensor power of a module $M$. It is easy to compute that the boundary operator $\delta: C^{n}(\pi, A) \longrightarrow C^{n+1}(\pi, A)$ is induced by the map $\partial: \mathbf{Z}(\pi)^{\otimes(n+2)} \longrightarrow \mathbf{Z}(\pi)^{\otimes(n+1)}$ defined by

$$
\begin{gathered}
\partial\left(x_{0} \otimes \cdots \otimes x_{n+1}\right)=\sum_{i=0}^{n}(-1)^{i} x_{0} \otimes \cdots \otimes x_{i} x_{i+1} \otimes \cdots \otimes x_{n+1} \\
+(-1)^{n+1} x_{0} \otimes \cdots \otimes x_{n}
\end{gathered}
$$

I claim that if you set $C_{n}(\pi)=\mathbf{Z}(\pi)^{\otimes(n+1)}$ with the boundary operator $\partial$, the resultant complex is a projective resolution of $\mathbf{Z}$ made into a $\pi$-module by having each element of $\pi$ act as the identity. Let us note that the action of $\pi$ on $\mathbf{Z}(G)^{\otimes(n+1)}$ is on the first coordinate, in accordance with the adjunction isomorphism above. Thus $\mathbf{Z}(\pi)^{\otimes(n+1)}=\mathbf{Z}(\pi) \otimes \mathbf{Z}(\pi)^{\otimes n}$ and $\mathbf{Z}(\pi)^{\otimes n}$ is just the free abelian group generated by $\pi^{n}$ and hence $\mathbf{Z}(\pi)^{\otimes(n+1)}$ is the free $\pi$-module generated by $\pi^{n}$. The augmentation $\mathbf{Z}(\pi) \longrightarrow \mathbf{Z}$ is the linear map that takes the elements of $\pi$ to the integer 1 . In order to show that $\operatorname{Ext}_{\pi}(\mathbf{Z}, M)$ is the Eilenberg-Mac Lane cohomology of $\pi$ with coefficients in $M$, it is sufficient to show that the augmented chain complex

$$
\cdots \longrightarrow \mathbf{Z}(\pi)^{\otimes(n+1)} \longrightarrow \mathbf{Z}(\pi)^{\otimes n} \longrightarrow \cdots \longrightarrow \mathbf{Z}(\pi) \longrightarrow \mathbf{Z} \longrightarrow 0
$$

is acyclic (that is, is an exact sequence), which implies that the unaugmented complex is a projective resolution of $\mathbf{Z}$ as a $\mathbf{Z}(\pi)$-module. We will actually show that the augmented complex is contractible as a complex of $\mathbf{Z}$-modules, although not as a complex of $\mathbf{Z}(\pi)$-modules. Define $s_{-1}: \mathbf{Z} \longrightarrow \mathbf{Z}(\pi)$ to be the linear map that sends 1 to $1 \in \pi$ (that is, the identity of $\pi$ ). For $n>0$, define $s_{n-1}: \mathbf{Z}(\pi)^{\otimes n}$ $\longrightarrow \mathbf{Z}(\pi)^{\otimes(n+1)}$ to be the linear map that takes $x_{1} \otimes \cdots \otimes x_{n}$ to $1 \otimes x_{1} \otimes \cdots \otimes x_{n}$. Then $\partial_{0} \circ s_{-1}=$ id clearly. For $n=1$, we have

$$
\left(\partial_{1} \circ s_{0}+s_{-1} \partial_{0}\right)(x)=\partial_{1}(1 \otimes x)+s_{-1}(1)=x-1+1=x
$$


For $n>1$,

$$
\begin{aligned}
\partial_{n} \circ s_{n-1}\left(x_{1} \otimes \cdots \otimes x_{n}\right)= & \partial_{n}\left(1 \otimes x_{1} \otimes \cdots \otimes x_{n}\right) \\
= & x_{1} \otimes \cdots \otimes x_{n} \\
& +\sum_{i=1}^{n-1}(-1)^{i}\left(1 \otimes x_{1} \otimes \cdots \otimes x_{i} x_{i+1} \otimes \cdots \otimes x_{n}\right) \\
& +(-1)^{n} 1 \otimes x_{1} \otimes \cdots \otimes x_{n-1}
\end{aligned}
$$

while

$$
\begin{gathered}
s_{n-2} \circ \partial_{n-1}\left(x_{1} \otimes \cdots \otimes x_{n}\right)=s_{n-2}\left(\sum_{i=1}^{n-1}(-1)^{i-1}\left(x_{1} \otimes \cdots \otimes x_{i} x_{i+1} \otimes \cdots \otimes x_{n}\right)\right) \\
+(-1)^{n-1} s_{n-2}\left(x_{1} \otimes \cdots \otimes x_{n-1}\right) \\
=\sum_{i=1}^{n-1}(-1)^{i-1}\left(1 \otimes x_{1} \otimes \cdots \otimes x_{i} x_{i+1} \otimes \cdots x_{n}\right) \\
+(-1)^{n-1} 1 \otimes x_{1} \otimes \cdots \otimes x_{n-1}
\end{gathered}
$$

and adding them up, we see that $\partial_{n} \circ s_{n-1}+s_{n-2} \circ \partial_{n-1}=$ id.

Thus it follows that the Eilenberg-Mac Lane cohomology groups of a group $\pi$ with coefficients in a $\pi$-module $A$ are just $\operatorname{Ext}_{\mathbf{Z}(\pi)}(\mathbf{Z}, A)$.

In the case of associative $K$-algebras, the development is similar. In this paragraph, we will denote by $\otimes$, the tensor product $\otimes_{K}$. A two-sided $\Lambda$-module is a left $\Lambda^{\mathrm{e}}=\Lambda \otimes \Lambda^{\mathrm{op}}$-module. The Hochschild cochain complex with coefficients in a module $A$ is shown to be the complex $\operatorname{Hom}_{\Lambda^{\mathrm{e}}}\left(C_{\bullet}, A\right)$ in which $C_{n}=\Lambda^{\otimes(n+2)}$ with boundary $\partial=\partial_{n}: C_{n} \longrightarrow C_{n-1}$ given by

$$
\partial\left(x_{0} \otimes \cdots \otimes x_{n+1}\right)=\sum_{i=0}^{n}(-1)^{i} x_{0} \otimes \cdots \otimes x_{i} x_{i+1} \otimes \cdots \otimes x_{n+1}
$$

By a similar formula to the group case, one shows that this chain complex augmented over $\Lambda$ is linearly (actually even right $\Lambda$-linearly) contractible and therefore a projective resolution of $\Lambda$ as a $\Lambda^{\mathrm{e}}$-module. It should be observed that $\Lambda \otimes \Lambda \cong \Lambda \otimes \Lambda^{\mathrm{op}}$ as a $\Lambda^{\mathrm{e}}$-module, although not, of course, as an algebra. The upshot is that the Hochschild cohomology of $\Lambda$ with coefficients in a two-sided $\Lambda$-module $A$ is simply $\operatorname{Ext}_{\Lambda^{\mathrm{e}}}(\Lambda, A)$.

The analysis of the Lie algebra case is somewhat more complicated (because the coboundary operator is more complicated) but the outcome is the same. If $\mathfrak{g}$ is a $K$-Lie algebra, the category of $\mathfrak{g}$-modules is equivalent to the category of left modules over the enveloping associative algebra $\mathfrak{g}^{\mathrm{e}}$. This is the quotient of the tensor algebra over the $K$-module underlying $\mathfrak{g}$ modulo the ideal generated by all $x \otimes y-y \otimes x-[x, y]$, for $x, y \in \mathfrak{g}$. Then one can show that the cochain complex comes from a projective resolution of $K$-with trivial $\mathfrak{g}$-action - so that the cohomology with coefficients in the $\mathfrak{g}$-module $A$ is $\operatorname{Ext}_{\mathfrak{g}^{\mathfrak{e}}}(K, A)$.

5.2 Comments. It was certainly elegant that Cartan and Eilenberg were able to find a single definition that gave cohomology theories of the previous decade in 
all three cases. Nonetheless there were three ad hoc features of their definitions that rendered their answer less than satisfactory:

1. what is a module;

2. what is $X^{e}$; and

3. what is $X_{J}$ ?

But the least satisfactory aspect of their definition did not become clear right away. It was those three cohomology theories ended up as the only ones for which the Cartan-Eilenberg theory was correct. This first showed up in Harrison's theory for commutative algebras.

\section{The Harrison cohomology theory}

Around 1960, Dave Harrison [1962] defined a cohomology theory for commutative algebras over a field. His original definition was rather obscure. As modified by the referee (who identified himself to Harrison as Mac Lane) it became somewhat less obscure, but still not obvious. Let $R$ ba commutative $K$-algebra with $K$ a field. If $0<i<n$ define a linear map $*: R^{\otimes i} \otimes R^{\otimes(n-i)} \longrightarrow R^{\otimes n}$ by

$$
\begin{gathered}
\left(x_{1} \otimes \cdots \otimes x_{i}\right) *\left(x_{i+1} \otimes \cdots \otimes x_{n}\right)=\left(x_{1} \otimes\left(x_{2} \otimes \cdots \otimes x_{i}\right) *\left(x_{i+1} \otimes \cdots \otimes x_{n}\right)\right) \\
+(-1)^{i}\left(x_{i+1} \otimes\left(x_{1} \otimes \cdots \otimes x_{i}\right) *\left(x_{i+2} \otimes \cdots \otimes x_{n}\right)\right)
\end{gathered}
$$

which together with

$$
\left(x_{1} \otimes \cdots \otimes x_{n}\right) *()=() *\left(x_{1} \otimes \cdots \otimes x_{n}\right)=\left(x_{1} \otimes \cdots \otimes x_{n}\right)
$$

defines inductively an operation on strings, called the shuffle. ${ }^{1}$ If $A$ a left $R$-module that is made into a two-sided $R$-module by having the same operation on both sides. Harrison defined a commutative $n$-cochain as an $f$ in the Hochschild cochain group $C^{n}(R, A)$ such that

$$
f\left(\left(x_{1} \otimes \cdots \otimes x_{i}\right) *\left(x_{i+1} \otimes \cdots \otimes x_{n}\right)\right)=0
$$

for all $0<i<n$ and all $\left(x_{1} \otimes \cdots \otimes x_{n}\right) \in R^{n}$. In other words, a commutative cochain was one that vanished on all proper shuffles. The commutative coboundary formula was the same as Hochschild's and it required a non-trivial argument to show that Harrison's cochains are invariant under it. The details are in his 1962 paper and can also be found in [Barr, 2002].

The coboundary of a 0-chain in $C(R, A)$-an element of $a \in A$ is the one chain $f$ for which $f(x)=a x-x a$. But if $A$ has the same action on both sides, this is 0 . Thus the cochain complex breaks up into two pieces, the degree 0 piece and the rest. Hence for Harrison, $H^{0}(R, A)=A$ and $H^{1}(R, A)=\operatorname{Der}(R, A)$, the group of derivations of $R$ to $A$. The groups $H^{2}(R, A)$ and $H^{3}(R, A)$ have the same interpretations as in the Hochschild theory, but restricted to the case that everything is commutative.

Harrison observed moreover that, provided the characteristic of the underlying ground field is not 2, the group of 2-cochains can be written as the direct sum of the commutative cochains and a complementary summand in such a way that the

\footnotetext{
${ }^{1}$ The reason it is called a shuffle is that it is the alternating sum of all possible ways of shuffling the first $i$ cards in an $n$ card deck with the remaining $n-i$. The inductive formula corresponds to the obvious fact that any shuffle can be thought of as consisting of taking the top card from one of the two decks, shuffling the remaining cards and then replacing that top card on top.
} 
Hochschild cohomology splits as the direct sum of two groups, one of which is the commutative cohomology and the other is a complementary term. In my Ph.D. thesis, [1962], I pushed this splitting up to degree 4, except that now characteristic 3 also had to be excluded. What I actually showed was the Hochschild cochain complex, truncated to degree 4, could be written as a direct sum of the Harrison cochain subcomplex and a complementary subcomplex and this splits the cohomology up to degree 4. (It might be thought that you need a splitting of the cochain complex up to degree 5 to do this, but that turns out to be unnecessary.) This was used to show various facts about the Harrison cohomology group of which the perhaps the most interesting was that when $R$ is a polynomial ring, then $H^{n}(R, A)=0$ for all $R$-modules $A$ and $n=2,3,4$. Actually, Harrison had shown this for $n=2,3$ in a different way, but it was not obvious how to extend his argument to higher dimension.

My proofs were highly computational and it was unclear how to extend any of it to higher dimensions. But I gnawed at it for five years and did eventually [Barr, 1968] find a relatively simple and non-computational construction of an idempotent in the rational group rings of symmetric groups that when applied to the Hochschild chain complex of an algebra over a field of characteristic 0 splits it into a commutative part and a complement, each invariant under the coboundary. This showed that when the ground field has characteristic 0, the Hochschild cohomology splits into two parts; one of the two is the Harrison cohomology and the other is a complement. Later, Gerstenhaber and Shack [1987], by discovering and factoring the characteristic polynomial of these idempotents, discovered a "Hodge decomposition" of the Hochschild groups:

$$
H^{n}(R, A)=\sum_{i=1}^{n} H^{n i}(R, A)
$$

where $H^{n 1}(R, A)$ is the Harrison group and the other summands are part of an infinite series whose $n$th piece vanishes in dimensions below $n$, so that in each degree the sum is finite.

Harrison's original paper also contained an appendix - written by me - that used the same ideas from Mac Lane's 1958 paper to deal with the case of a commutative algebra over a general coefficient ring. However, the referee - who identified himself to Harrison as Mac Lane - insisted on an appendectomy and the paper is now lost. From this, I conclude that Mac Lane felt the approach was likely a dead end and I tend to agree.

6.1 Does the Harrison cohomology fit the Cartan-Eilenberg model? As I have already hinted, Harrison was not a fan of the Cartan-Eilenberg model of what a cohomology theory was. I do not recall that he ever mentioned it, I do not think I was even aware of it before arriving at Columbia in the fall of 1962 and I do not know if Harrison had ever considered whether his theory fit into it. It is a bit odd, since it seems clear that Cartan and Eilenberg believed that their book defined what a cohomology theory was. They scarcely mentioned the older definitions in their book and, as far as I can tell (the book lacks a bibliography), they did not even cite either Eilenberg-Mac Lane paper [1947a,b]. But Harrison's definition was in the style of the older definitions. To put his theory into the Cartan-Eilenberg framework, there would have to be, for each commutative ring $R$, an enveloping algebra $R^{e}$ such that left $R^{e}$-modules were the same as left $R$-modules and a module 
$R_{J}$ for which $H^{n}(R, A)=\operatorname{Ext}_{R^{e}}^{n}\left(R_{J}, A\right)$. Since left $R$-modules were the same as left $R^{e}$-modules, we would have to take $R^{e}$ to be $R$ or something Morita equivalent to it. But Ext is invariant under Morita equivalence so nothing can be gained by using anything but $R$. As for $R_{J}$, we leave that aside for the moment and point out that one consequence of the cohomology being an Ext is that it vanishes whenever $A$ is injective. This is true for cohomology theories of groups, associative algebras, and Lie algebras, but is not true for commutative algebras. The easiest example is the following. Let $K$ be any field. It is easy to see (and well-known) that the algebra of dual numbers $R=K[x] /\left(x^{2}\right)$ is self-injective. On the other hand, we have the non-split exact sequence

$$
0 \longrightarrow x^{2} \cdot K[x] / x^{4} \longrightarrow K[x] /\left(x^{4}\right) \longrightarrow K[x] /\left(x^{2}\right) \longrightarrow 0
$$

of commutative rings, whose kernel is, as an $R$-module, isomorphic to $R$ and hence is injective. Thus $H^{2}(R, R) \neq 0$. See [Barr, 1968a]. This example doomed the attempted redefinition of commutative cohomology that appeared in [Barr, 1965a, 1965b].

Here is an explanation of what goes wrong. If $R$ is a commutative $K$-algebra, then the chain complex that has $R^{\otimes(n+2)}$ with the Hochschild boundary operator, is a projective resolution of $R$ as an $R \otimes R$-module. If $A$ is a symmetric module and $B$ is any two-sided $R$-module, it is evident that

$$
\operatorname{Hom}_{R \otimes R}(B, A) \cong \operatorname{Hom}_{R}\left(R \otimes_{R \otimes R} B, A\right)
$$

since $R \otimes_{R \otimes R} B$ is just the symmetrization of $B$. Since $R \otimes_{R \otimes R} R^{\otimes(n+2)}=R^{\otimes(n+1)}$, the Hochschild cochain complex of $R$ with coefficients in $A$ is

$$
\operatorname{Hom}_{R \otimes R}\left(R^{\otimes(n+2)}, A\right) \cong \operatorname{Hom}_{R}\left(R^{\otimes(n+1)}, A\right)
$$

It follows that the symmetrized chain complex is not generally acyclic; its homology is $\operatorname{Tor}^{R \otimes R}(R, R)$ and that is trivial if and only if $R$ is separable. Even if it is, the quotient complex modulo the shuffles will not be generally acyclic.

\section{Cohomology as a functor in the first argument}

Although Harrison had an 8 term exact sequence involving the cohomology of $R$, that of $R / I$ for an ideal $I$ and $\operatorname{Ext}_{R}(I,-)$, the functoriality of cohomology in its first argument had been mostly ignored. If a connected series of functors is to look like a derived functor in a contravariant variable, it should vanish in all positive dimensions when that variable is free. But cohomology did not vanish when its first variable was free. In fact, when the group, algebra, or Lie algebra is free, the cohomology vanishes in dimensions greater than 1 . This is also the case for commutative algebras, but only in characteristic 0 . This, as well as other indications, suggested that if one wanted to view cohomology as a functor in the first variable, it would be best to drop the lowest degree term, renumber all the rest by -1 and also change the new lowest degree term from derivations modulo inner derivations to simply derivations. Thus was born the idea of cohomology as the derived functor of derivations, see [Barr and Rinehart, 1964].

Let us call this cohomology with the lowest term dropped and the next one modified the dimension-shifted cohomology. For the commutative cohomology, as already noted, there are no inner derivations and the lowest degree term is merely the coefficient module, so dropping it entails no loss of information. For the others, there is some cost. For associative and Lie algebras the cohomology vanishes in 
all dimensions if and only if the algebra is (finite-dimensional and) separable. For other purposes, the dimension-shifted cohomology seems better. For instance, the Stallings-Swan theorem states that a group is free if and only if its cohomological dimension is 1 . Using the dimension-shifted theory, that number changes to 0 , which is what you might expect for projectives (of course, every projective group is free).

So the first definition of the dimension-shifted is simply apply the functor $\operatorname{Der}(X,-)$ (here $X$ is the group or algebra or whatever) to the category of $X$ modules and form the derived functor. This means, given a module $A$ find an injective resolution

$$
0 \longrightarrow Q_{0} \longrightarrow Q_{1} \longrightarrow \cdots \longrightarrow Q_{n} \longrightarrow \cdots
$$

apply $\operatorname{Der}(X,-)$ to get the chain complex

$$
0 \longrightarrow \operatorname{Der}\left(X, Q_{0}\right) \longrightarrow \operatorname{Der}\left(X, Q_{1}\right) \longrightarrow \cdots \longrightarrow \operatorname{Der}\left(X, Q_{n}\right) \longrightarrow \cdots
$$

and define the cohomology groups to be the cohomology of that cochain complex. The trouble with this definition is that it automatically makes the cohomology vanish when the coefficients are injective and therefore can represent the cohomology only for theories that vanish on injective coefficients.

Before turning to other theories, there is one more point to be made. Since Der is like a homfunctor, it is quite easy to see that it preserves limits. The special adjoint functor theorem implies that it is representable by an object we call Diff (for module of differentials, since differentials are dual to derivations). The map $A \longrightarrow \operatorname{Der}(X, A)$ that sends an element of $A$ to the inner derivation at $A$ is the component at $A$ of a natural transformation $\operatorname{Hom}_{X^{e}}\left(X^{e},-\right) \longrightarrow \operatorname{Hom}_{X^{e}}$ (Diff, - ) and is thereby induced by a homomorphism Diff $\longrightarrow X^{e}$. In the three classic cases of groups, associative algebras and Lie algebras, this homomorphism is injective and the quotient module $X^{e} /$ Diff is the heretofore mysterious $X_{J}$. Thus in those three cases, not only is the cohomology $\operatorname{Ext}_{X^{e}}\left(X_{J}, A\right)$, but the dimension-shifted cohomology is $\operatorname{Ext}_{X^{e}}(\mathrm{Diff}, A)$. Thus we have removed one of the ad hoc features from the Cartan-Eilenberg theory. It can be replaced by a question as to why the map from Diff $\longrightarrow X^{e}$ is injective in those cases, but at least we know where $X_{J}$ comes from. The second special item is $X^{e}$ but $X^{e}$ is determined, up to Morita equivalence, by the fact that $X^{e}$-modules are the same as $X$-modules. Since Ext is invariant under Morita equivalence, that ambiguity is not important. The third $a d$ hoc feature of the Cartan-Eilenberg definition is the definition of module and we will explain Jon Beck's surprising and elegant answer to that question next.

\section{Beck modules}

8.1 The definition of Beck modules. We begin Beck's theory by looking at an example in some detail. Denote by Grp the category of groups. If $\pi$ is a group, the category Grp/ $\pi$ has as objects group homomorphisms $p: \Pi \longrightarrow \pi$. If $p^{\prime}: \Pi^{\prime} \longrightarrow \pi$ is another object, a map $f: p^{\prime} \longrightarrow p$ is a commutative triangle

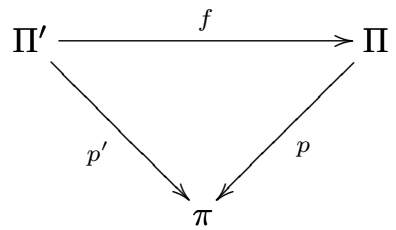


Given a $\pi$-module $A$, denote by $A \rtimes \pi$ the extension corresponding to the 0 element of $H^{2}(\pi, A)$. This means the underlying set is $A \times \pi$ and the multiplication is given by $(a, x)(b, y)=(a+x b, x y)$. The second coordinate projection makes $A \rtimes \pi$ into an object of $\mathrm{Grp} / \pi$.

Let us calculate the set $\operatorname{Hom}_{\mathrm{Grp} / \pi}(\Pi \stackrel{p}{\rightarrow} \pi, A \rtimes \pi \rightarrow \pi)$. A homomorphism $f: \Pi$ $\longrightarrow A \rtimes \pi$ is a function $\Pi \longrightarrow A \times \pi$. But in order to be a morphism in the category, the second coordinate must be $p$. If we call the first coordinate $d$, then $f=(d, p)$. In order to be a group homomorphism, we must have, for any $x, y \in \Pi$ that

$$
(d(x y), p(x y))=(d(x), p(x))(d(y), p(y))=(d(x)+p(x) d(y), p(x) p(y))
$$

Since $p$ is already a homomorphism, the second coordinates are equal. As for the first coordinates, the required condition is that $d(x y)=p(x) d(y)+d(x)$. Except for the $p$ on the right hand side, this is just the formula that defines a derivation. But we can use $p$ to make any $\pi$-module into a $\Pi$-module and then this condition is just that $d$ is a derivation of $\Pi$ to $A$. Thus we have shown that

$$
\operatorname{Hom}_{\mathrm{Grp} / \pi}(\Pi \stackrel{p}{\rightarrow} \pi, A \rtimes \pi \rightarrow \pi)=\operatorname{Der}(\Pi, A)
$$

Evidently, $\operatorname{Der}(\Pi, A)$ is an abelian group from the additive structure of $\mathrm{A}$ and it is easy to see that a morphism $\left(\Pi^{\prime} \rightarrow \pi\right) \longrightarrow(\Pi \rightarrow \pi)$ induces not merely a function but a group homomorphism $\operatorname{Der}(\Pi, A) \longrightarrow \operatorname{Der}\left(\Pi^{\prime}, A\right)$. This means that $A \rtimes \pi \rightarrow \pi$ is actually an abelian group object in $\mathrm{Grp} / \pi$.

The converse is also true. It is a standard fact about abelian group objects in categories with finite products that a group object $G$ is given by a global section $1 \longrightarrow G$, an inverse map $G \longrightarrow G$ and a multiplication $G \times G \longrightarrow G$ and that the multiplication map is just the product of the two projections. If $p: \Pi \longrightarrow \pi$ is a group object in Grp/ $\pi$, the terminal object of the category is id $: \pi \longrightarrow \pi$ and thus the zero map is just a homomorphism $\pi \longrightarrow \Pi$ that splits $p$. If $K$ is the kernel of $p$, it is a normal subgroup and we will write its group operation as + and its identity element as 0 even though we have not yet proved it commutative. By extending the notation to the non-commutative case, we can write $\Pi=K \rtimes \pi$. In this notation, the zero morphism is given by $z(x)=(0, x)$. The product in the category Grp $/ \pi$ is just the fibered product over $\pi$. Since $\Pi \times_{\pi} \Pi=K \times K \rtimes \pi$, the multiplication is a homomorphism

$$
m: K \times K \rtimes \pi \longrightarrow K \rtimes \pi
$$

over $\pi$. We can write this as $m(a, b, x)=(f(a, b, x), x)$ for $a, b \in K$ and $x \in K$. Since $m$ preserves the identity of the group object, $m(0,0, x)=(0, x)$ from which it follows that $f(0,0, x)=0$ for any $x \in \pi$. But then

$$
\begin{aligned}
m(a, b, x) & =m(a, b, 1) m(0,0,1)=(f(a, b, 1), 1)(f(0,0, x), x) \\
& =(f(a, b, 1), 1)(0, x)=(f(a, b, 1), x)
\end{aligned}
$$

so that $f$ does not depend on $x$. Write $f(a, b, 1)=a * b$. Then since $(0, x)$ is the identity in the fiber over $x, m(a, 0, x)=(a, x)=m(0, a, x)$ so that $a * 0=0 * a=a$. But then

$$
\begin{aligned}
m(a, b, x) & =m(a, 0,1) m(0, b, 1) m(0,0, x)=(a * 0,1)(0 * b, 1)(0, x) \\
& =(a * 0+0 * b, x)=(a+b, x)
\end{aligned}
$$


while at the same time

$$
\begin{aligned}
m(a, b, x) & =m(0, b, 1) m(a, 0,1) m(0,0, x)=(0 * b, 1)(a * 0,1)(0, x) \\
& =(0 * b+a * 0, x)=(b+a, x)
\end{aligned}
$$

from which we conclude that $*=+$ and is commutative. The action of $\Pi$ on $K$ is by conjugation and since $K$ is commutative, this induces an action of $\pi$ on $K$. If $q: \Gamma \longrightarrow \pi$ is a group over $\pi$, then a morphism $f:(\Gamma \rightarrow \pi) \longrightarrow(\Pi \rightarrow \pi)$ has the form $f(y)=(d y, q y)$ and to be a homomorphism we must have

$$
\begin{aligned}
\left(d\left(y y^{\prime}\right), q\left(y y^{\prime}\right)\right) & =f\left(y y^{\prime}\right)=f(y) f\left(y^{\prime}\right)=(d y, q y)\left(d y^{\prime}, q y^{\prime}\right) \\
& =\left(d y+(q y)\left(d y^{\prime}\right),(q y)\left(q y^{\prime}\right)\right)
\end{aligned}
$$

which means that $d\left(y y^{\prime}\right)=d y+(q y)\left(d y^{\prime}\right)$ which is the definition of a derivation (with respect to $q$ ).

This is one case of:

Theorem 2 (Beck) Let $\mathscr{X}$ be one of the familiar categories (groups, algebras and rings, Lie algebras, commutative algebras and rings, Jordan algebras, ...). Then the category of modules over an object $X$ of $\mathscr{X}$ is (equivalent to) the category of abelian group objects in the category $\mathscr{X} / X$. Moreover, let $A$ be an $X$-module with $Y \longrightarrow X$ the corresponding abelian group object in $\mathscr{X} / X$. Then for an object $Z \longrightarrow X$ of that category, $\operatorname{Hom}(Z \rightarrow X, Y \rightarrow X)$ is canonically isomorphic to $\operatorname{Der}(Z, A)$.

The upshot of this result is that not only do we know what a module is for an object of any category, we also know what a derivation into that module is. For example, a module over the set $I$ is just an $I$-indexed family $A=\left\{A_{i} \mid i \in I\right\}$ of abelian groups and $\operatorname{Der}(I, A)=\prod_{i \in I} A_{i}$. For any $X \longrightarrow I$, which is just an $I$-indexed family $\left\{X_{i} \mid i \in I\right\}$, one can see that $\operatorname{Der}(X, A)=\prod_{i \in I} A_{i}^{X_{i}}$.

After I gave my talk, I had a private discussion with Myles Tierney and Alex Heller. Heller remarked that he first heard the definition of Beck module from Eilenberg and wondered whether the definition was originally his. Tierney, who was a student of Eilenberg's at the same time as Beck, recalled that it taken a couple months for Beck to convince Eilenberg of the correctness of his definition, but that once convinced, Eilenberg embraced it enthusiastically.

As an aside, let me say that this is the definition of bimodule. One-sided modules cannot be got as special cases of this and are apparently a different animal. Apparently one-sided modules are part of representation theory and two-sided modules are part of extension theory.

\section{Enter triples}

Triples (also known as monads) have been used in many places and for many reasons in category theory (as well as in theoretical computer science, especially in the theory of datatypes). They were originally invented (by Godement in [1958]) for the purpose of describing standard flabby resolutions of sheaves. They were being used for this purpose by Eckmann and his students around 1960 and by Eilenberg and Moore in their Memoir [1965a]. But this was always in additive categories (although the triples were not always additive, but Eilenberg and Moore assumed not only that but that they preserved kernels, although neither hypothesis was necessary for their purposes). 
Beck was the first person who used a triple (more precisely, a cotriple) in a non-additive category to define a cohomology theory. Once more, I will illustrate what he did in the category of groups.

9.1 Triples and Eilenberg-Moore algebras. The definitions are available in many places and so I will just give a rapid sketch. If $\mathscr{X}$ is a category, a triple $\mathbf{T}$ on $\mathscr{X}$ consists of $(T, \eta, \mu)$ where $T$ is an endofunctor on $\mathscr{X}$ and $\eta$ and $\mu$ are natural transformations: $\eta: \mathrm{Id} \longrightarrow T$ and $\mu: T^{2} \longrightarrow T$ such that the diagrams
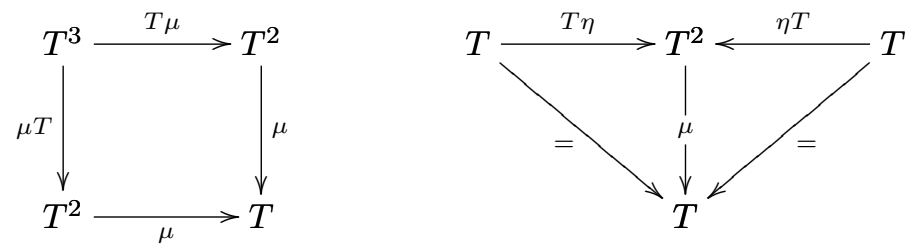

commute. A cotriple is a triple in the dual category. Hence a cotriple $\mathbf{G}$ consists of $(G, \epsilon, \delta)$ with $G$ an endofunctor and $\epsilon: G \longrightarrow$ Id and $\delta: G \longrightarrow G^{2}$ natural transformations such that the dual of the above diagrams commute. Given an adjoint pair $F: \mathscr{X} \longrightarrow \mathscr{Y}$ left adjoint to $U: \mathscr{Y} \longrightarrow \mathscr{X}$, with adjunction arrows $\eta: \mathrm{Id} \longrightarrow U F$ and $\epsilon: F U \longrightarrow \mathrm{Id}$, then $(U F, \eta, U \epsilon F)$ is a triple on $\mathscr{X}$ and $(F U, \epsilon, F \eta U)$ is a cotriple on $\mathscr{Y}$. Peter Huber (a student of Eckmann's) told me once that they had proved this theorem - a simple argument with naturality - because they were having so much trouble verifying the commutations in the diagram above and noticed that all their triples and cotriples were associated with adjoint pairs (see [Huber, 1961]). It is much easier to verify an adjunction than a triple. The converse is also true; every (co-)triple does arise in this way from an adjoint pair. There are two distinct proofs of this; due to Kleisli [1964, 1965] and EilenbergMoore [1965b]. For most purposes in algebra, the Eilenberg-Moore algebras are more interesting. But triples have also entered theoretical computer science and there the Kleisli construction is the main one.

9.2 Beck cohomology. As above, it is convenient to illustrate Beck's results in the category of groups. The adjoint pair $F \multimap U$ where $F$ : Set $\longrightarrow$ Grp and $U: \operatorname{Grp} \longrightarrow$ Set is the underlying set functor, gives rise to a cotriple on Grp as described. If $\pi$ is a group, then $G \pi$ is the free group on the underlying set of $\pi$. As with any cotriple, there results a functor that assigns to each group $\pi$ a simplicial group that has $G^{n+1} \pi$ in degree $n$. The face operators are constructed from $\epsilon$ by $d^{i}=d_{n}^{i}=G^{i} \epsilon G^{n-i}: G^{n+1} \longrightarrow G^{n}$ and $s^{i}=s_{n}^{i}=G^{i} \delta G^{n-i}: G^{n+1}$ $\longrightarrow G^{n+2}$. The naturality and the commuting diagrams satisfied by a cotriple imply the simplicial identities:

$$
\begin{aligned}
& d_{n}^{i} \circ d_{n+1}^{j}=d_{n}^{j-1} \circ d_{n+1}^{i} \quad \text { if } 0 \leq i<j \leq n+1 \\
& s_{n}^{j} \circ s_{n-1}^{i}=s_{n}^{i} \circ s_{n-1}^{j-1} \quad \text { if } 0 \leq i<j \leq n \\
& d_{n+1}^{i} \circ s_{n}^{j}= \begin{cases}s_{n-1}^{j-1} \circ d_{n}^{i} & \text { if } 0 \leq i<j \leq n \\
1 & \text { if } 0 \leq i=j \leq n \text { or } 0 \leq i-1=j<n \\
s_{n-1}^{j} \circ d_{n}^{i-1} & \text { if } 0<j<i-1 \leq n\end{cases}
\end{aligned}
$$

We now form, for each object $X$ and each $X$-module $A$, the cochain complex

$$
0 \longrightarrow \operatorname{Der}(G X, A) \longrightarrow \cdots \longrightarrow \operatorname{Der}\left(G^{n} X, A\right) \longrightarrow \operatorname{Der}\left(G^{n+1} X, A\right) \longrightarrow \cdots
$$


with the coboundary map

$$
\sum_{i=0}^{n}(-1)^{i} \operatorname{Der}\left(d^{i} X, A\right): \operatorname{Der}\left(G^{n} X, A\right) \longrightarrow \operatorname{Der}\left(G^{n+1} X, A\right)
$$

Curiously, the $\delta$ of the cotriple plays no part in this, but it is absolutely necessary in applying the acyclic models theorem mentioned below.

This gives a uniform treatment for cohomology in any equational category. Beck also showed that the group $H^{1}(X, A)$ did classify extensions of $X$ with kernel $A$, as expected. He did not explore the second cohomology that in known cases was relevant to extensions with non-abelian kernels. Later I did in a couple cases and discovered some problems in the general case that showed that the interpretation of the dimension-shifted $H^{2}$ could not be quite the same as in the known cases (Jordan algebras supplied the first counter-example).

\section{Comparison theorems}

Jon Beck and I started thinking in early 1964 about the connection between the various cohomology theories that had been invented in an ad hoc way and the Beck (or cotriple) cohomology theories. Let us assume that we are talking about the dimension-shifted versions of the former. So we know that $H^{0}(X, A)=$ $\operatorname{Der}(X, A)$ for both theories and that both versions of $H^{1}(X, A)$ classify the same set of extensions and hence that they are at least isomorphic (although naturality was still an issue). But we had no idea whatever about $H^{2}$ or any higher dimension. The complexes look entirely different. The Eilenberg-Mac Lane complex has, in degree $n$, functions of $n+1$ (because of the dimension shift) variables from $\pi$ to $A$, while the cotriple complex has functions of one variable from $G^{n+1} \pi$ to $A$. We spent the fall term of 1964 working on this problem and getting exactly nowhere. For group cohomology, one approach would have been to show that the chain complex

$$
\cdots \longrightarrow \text { Diff } G^{n+1} \pi \longrightarrow \text { Diff } G^{n} \pi \longrightarrow \cdots \longrightarrow \text { Diff } G \pi \longrightarrow \text { Diff } \pi \longrightarrow 0
$$

with boundary operator $\sum_{i=1}^{n}(-1)^{n} \operatorname{Diff} d^{i}$, is exact; for then the positive part of that complex would have been a projective resolution of Diff $\pi$. In retrospect, we now know how to do that directly. Fortunately for us we did not find that argument for it would have been a dead end.

Instead, Beck spoke to Harry Appelgate during the term break between 1964 and 1965. Appelgate suggested trying to solve the problem by using acyclic models, one of the subjects of his thesis [1965]. We did and within a few days we had solved the comparison problem for groups and associative algebras. For some reason, we never looked at Lie algebras, while commutative algebras, for the time being, resisted our methods.

The case of commutative algebras was settled in the late 1960s. In finite characteristic, Harrison's definition was not equivalent to the cotriple cohomology, as shown by an example due to Michel André. In characteristic 0, it was shown in [Barr, 1968] that the two theories coincided. It is shown in [Barr, 1996] that the same is true for Lie algebras.

10.1 Acyclic models. Here is a brief description of the acyclic models theorem that we proved and used to compare the Cartan-Eilenberg cohomology with the cotriple cohomology. The context of the theorem is a category $\mathscr{X}$ equipped with a cotriple $\mathbf{G}=(G, \epsilon, \delta)$ and an abelian category $\mathscr{A}$. We are given augmented 
chain complex functors $K_{\bullet}=\left\{K_{n}\right\}, L_{\bullet}=\left\{L_{n}\right\}: \mathscr{X} \longrightarrow \mathscr{A}$, for $n \geq-1$. We say that $K_{\bullet}$ is $G$-presentable if there is a natural transformation $\theta_{n}: K_{n} \longrightarrow K_{n} G$ for all $n \geq 0$ (note: not for $n=-1$ ) such that $K_{n} \epsilon \circ \theta_{n}=$ id for all $n \geq 0$. We say that $L_{\bullet}$ is $G$-contractible if the complex $L_{\bullet} G \longrightarrow 0$ has a natural contracting homotopy (which is called $s$ below).

Theorem 3 Suppose that $K_{\bullet}$ is $G$-presentable and $L_{\bullet}$ is $G$-contractible. Then any natural transformation $f_{-1}: K_{-1} \longrightarrow L_{-1}$ can be extended to a natural transformation $f_{\bullet}: K_{\bullet} \longrightarrow L_{\bullet}$. Any two extensions of $f_{-1}$ are naturally homotopic.

Proof Here are the two diagrams required for the proof. The computations are straightforward. The map $f_{n}$ is defined as the composite,

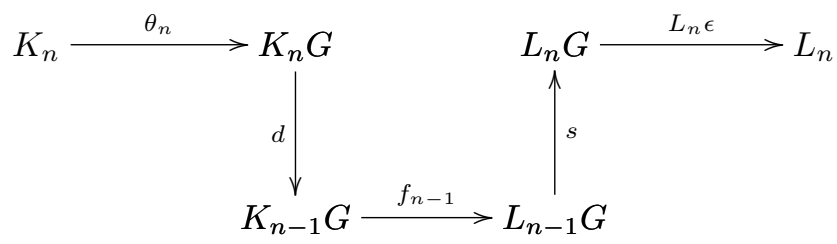

while the $n$th homotopy is defined as the difference of the upper and lower composite in,

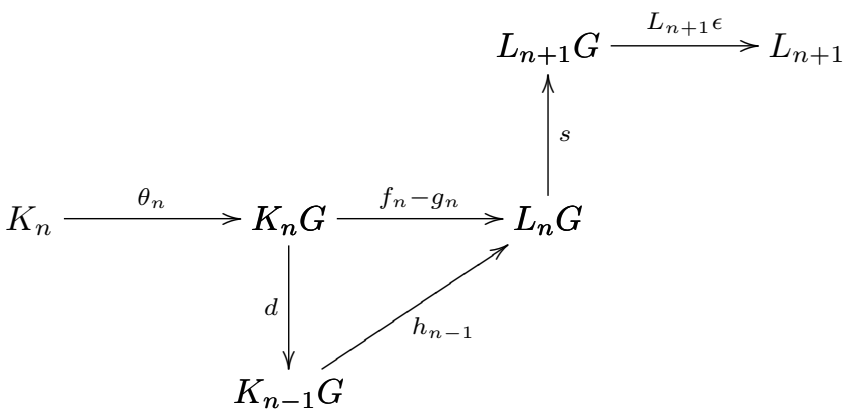

Of course, an immediate consequence of this is that if $K_{\bullet}$ and $L_{\bullet}$ each satisfy both hypotheses and $K_{-1}$ is naturally isomorphic to $L_{-1}$, then $K_{\bullet}$ is naturally homotopic to $L_{\bullet}$. This result is augmented by

Proposition 1 Suppose that $L_{n}=L_{-1} G^{n+1}$ with coboundary

$$
\sum_{i=0}^{n}(-1)^{i} G^{i} \epsilon G^{n-i}
$$

Then $L_{\bullet}$ is both $G$-presentable and $G$-contractible.

In fact, $\delta$ is used to show both claims.

Before describing the modern acyclic models theorem that can be used to prove this, we will describe the issues involved. Basically, two things are required to show that a cochain complex functor is cohomologous to the cotriple complex. The first seems somewhat odd at first glance, but is satisfied in examples. That is that the terms of the complex do not depend on the structure of the object in question, but only on the underlying set (or module). It is a curious fact, but obvious from the definition that the structure is used only in the definition of the coboundary homomorphism. The second condition required is that the cohomology 
of a free object should vanish in positive degrees. This condition is automatic for the cotriple cohomology; hence this is a necessary condition if the two theories are to be equivalent. This is where the problem arises for Harrison's commutative cohomology in finite characteristic. Michel André showed that in characteristic $p$ there was a non-zero cohomology class in degree $2 p-1$.

Here is the argument for acyclicity in the case of free groups. We begin by observing that a free group is also free as far as derivations is concerned.

Proposition 2 Suppose that $\Pi$ is free on basis $X$ and $M$ is a $\Pi$-module. Then any function $\tau: X \longrightarrow M$ extends to a unique derivation $\Pi \longrightarrow M$.

Proof Let $U:$ Grp $\longrightarrow$ Set denote the underlying functor. This result follows from the sequence of isomorphisms

$$
\begin{aligned}
\operatorname{Der}(\Pi, M) & \cong \operatorname{Hom}_{\mathrm{Grp} / \pi}(\Pi \rightarrow \pi, M \rtimes \pi \rightarrow \pi) \\
& \cong \operatorname{Hom}_{\mathrm{Set} / U \pi}(X \rightarrow U \pi, U M \times U \pi \rightarrow U \pi) \\
& \cong \operatorname{Hom}_{\mathrm{Set}}(X, U M)
\end{aligned}
$$

This implies that Diff( $\Pi)$ is the free $\pi$-module generated by $S$.

It is not hard to show that $C_{\bullet}$ is an exact chain complex and hence $C_{\bullet}(\Pi)$ is a free resolution of $\operatorname{Diff}(\Pi)$. In the case that $\Pi$ is free, this is then a free resolution of a free module and hence necessarily split. However, we would rather get the extra information available if we know that the splitting is natural, namely that we then get a homotopy equivalence between the two chain complex functors.

We start by defining a homomorphism $\partial: C_{0}(\Pi) \longrightarrow \operatorname{Diff}(\Pi)$. There is a function $\tau: X \longrightarrow \operatorname{Diff}(\Pi)$ which is the inclusion of the basis. This extends to a derivation $\tau: \Pi \longrightarrow \operatorname{Diff}(\Pi)$ as above. Since $C_{0}(\Pi)$ is freely generated by the elements of $\Pi$, this derivation $\tau$ extends to a $\pi$-linear function $\partial: C_{0}(\Pi) \longrightarrow \operatorname{Diff}(\Pi)$. In accordance with the recipe above, $\partial$ is defined on elements of $\Pi$ recursively as follows. We will denote by $\langle w\rangle$ the basis element of $C_{0}(\Pi)$ corresponding to $w \in \Pi$. As above, either $w=1$ or $w=x v$ or $w=x^{-1} v$ for some $x \in X$ and some $v \in \Pi$ shorter than $w$. Then

$$
\partial\langle w\rangle= \begin{cases}x \partial\langle v\rangle+x & \text { if } w=x v \\ x^{-1} \partial\langle v\rangle-x^{-1} x & \text { if } w=x^{-1} v \\ 0 & \text { if } w=1\end{cases}
$$

Now define $s: \operatorname{Diff}(\Pi) \longrightarrow C_{0}(\Pi)$ to be the unique $\pi$-linear map such that $s(d x)=$ $\langle x\rangle$ for $x \in X$. Since Diff(ח) is freely generated by all $d x$ for $x \in X$, this does define a unique homomorphism. For $x \in X$, we have that $\partial \circ s(d x)=\partial\langle x\rangle=d x$ and so $\partial \circ s=$ id.

For each $n \geq 0$ we define a homomorphism $s: C_{n} \longrightarrow C_{n+1}$ as follows. We know that $C_{n}$ is the free $\pi$-module generated by $\Pi^{n+1}$. We will denote a generator by $\left\langle w_{0}, \cdots, w_{n}\right\rangle$ where $w_{0}, \ldots, w_{n}$ are words in elements of $X$ and their inverses. Then we define $s: C_{n} \longrightarrow C_{n+1}$ by induction on the length of the first word:

$$
s\left\langle w_{0}, \ldots, w_{n}\right\rangle= \begin{cases}x s\left\langle w, w_{1}, \ldots, w_{n}\right\rangle-\left\langle x, w, w_{1}, \ldots\right\rangle & \text { if } w_{0}=x w \\ x^{-1} s\left\langle w, w_{1}, \ldots, w_{n}\right\rangle+x^{-1}\left\langle x, w_{0}, w_{1}, \ldots\right\rangle & \text { if } w_{0}=x^{-1} w \\ \left\langle 1,1, w_{1}, \ldots, w_{n}\right\rangle & \text { if } w_{0}=1\end{cases}
$$

Proposition 3 For any word $w$ and any $x \in X$

$$
s\left\langle x w, w_{1}, \ldots, w_{n}\right\rangle=x s\left\langle w, w_{1}, \ldots, w_{n}\right\rangle-\left\langle x, w, w_{1}, \ldots, w_{n}\right\rangle
$$




$$
s\left\langle x^{-1} w, w_{1}, \ldots, w_{n}\right\rangle=x^{-1} s\left\langle w, w_{1}, \ldots, w_{n}\right\rangle+\left\langle x^{-1}, w_{0}, w_{1}, \ldots, w_{n}\right\rangle
$$

Proof These are just the recursive definitions unless $w$ begins with $x^{-1}$ for the first equation or with $x$ for the second. Suppose $w=x^{-1} v$. Then from the definition of $s$,

$$
s\left\langle w, w_{1} \ldots, w_{n}\right\rangle=x^{-1} s\left\langle v, w_{1}, \ldots, w_{n}\right\rangle+x^{-1}\left\langle x, w, w_{1}, \ldots, w_{n}\right\rangle
$$

so that

$$
\begin{aligned}
s\left\langle x w, w_{1}, \ldots, w_{n}\right\rangle & =s\left\langle v, w_{1}, \ldots, w_{n}\right\rangle \\
& =x s\left\langle w, w_{1}, \ldots, w_{n}\right\rangle-\left\langle x, w, w_{1}, \ldots, w_{n}\right\rangle
\end{aligned}
$$

The second one is proved similarly.

Now we can prove that $s$ is a contraction. First we will do this in dimension 0 , then, by way of example, in dimension 2 ; nothing significant changes in any higher dimension. In dimension 0 , suppose $w$ is a word and we suppose that for any shorter word $v$, we have that $s \circ \partial\langle v\rangle+\partial \circ s\langle v\rangle=\langle v\rangle$. If $x=1$, then

$$
s \circ \partial\langle 1\rangle+\partial \circ s\langle 1\rangle=\partial\langle 1,1\rangle=1\langle 1\rangle-\langle 1\rangle+\langle 1\rangle=\langle 1\rangle
$$

If $w=x v$, with $x \in X$, then

$$
\begin{aligned}
\partial \circ s\langle w\rangle+s \circ \partial\langle w\rangle & =\partial(x s\langle v\rangle-\partial\langle x, v\rangle)+s(d w) \\
& =x \partial \circ s\langle v\rangle-x\langle v\rangle+\langle x v\rangle-\langle x\rangle+s(x \partial(v)+d x) \\
& =\langle w\rangle+x(\partial \circ s+s \circ \partial-1)\langle v\rangle-\langle x\rangle+\langle x\rangle=\langle w\rangle
\end{aligned}
$$

A similar argument takes care of the case that $w=x^{-1} v$. In dimension 2 , the chain group $C_{2}(\Pi)$ is freely generated by $\Pi^{3}$. If we denote a generator by $\left\langle w_{0}, w_{1}, w_{2}\right\rangle$, we argue by induction on the length of $w_{0}$. If $w_{0}=1$, then

$$
\begin{aligned}
s \circ \partial\left\langle 1, w_{1}, w_{2}\right\rangle & =s\left(\left\langle w_{1}, w_{2}\right\rangle-\left\langle w_{1}, w_{2}\right\rangle+\left\langle 1, w_{1} w_{2}\right\rangle-\left\langle 1, w_{1}\right\rangle\right) \\
& =\left\langle 1,1, w_{1} w_{2}\right\rangle-\left\langle 1,1, w_{1}\right\rangle
\end{aligned}
$$

while

$$
\begin{aligned}
\partial \circ s\left\langle 1, w_{1}, w_{2}\right\rangle & =\partial\left(\left\langle 1,1, w_{1}, w_{2}\right\rangle\right) \\
& =\left\langle 1, w_{1}, w_{2}\right\rangle-\left\langle 1, w_{1}, w_{2}\right\rangle+\left\langle 1, w_{1}, w_{2}\right\rangle-\left\langle 1,1, w_{1} w_{2}\right\rangle+\left\langle 1,1, w_{1}\right\rangle
\end{aligned}
$$

and these add up to $\left\langle 1, w_{1}, w_{2}\right\rangle$. Assume that $(\partial \circ s+s \circ \partial)\langle w\rangle=\langle w\rangle$ when $w$ is shorter than $w_{0}$. Then for $w_{0}=x w$,

$$
\begin{aligned}
\partial \circ s\left\langle x w, w_{1}, w_{2}\right\rangle= & x \partial \circ s\left\langle w, w_{1}, w_{2}\right\rangle-\partial\left\langle x, w, w_{1}, w_{2}\right\rangle \\
= & x \partial \circ s\left\langle w, w_{1}, w_{2}\right\rangle-x\left\langle w, w_{1}, w_{2}\right\rangle+\left\langle x w, w_{1}, w_{2}\right\rangle \\
& -\left\langle x, w w_{1}, w_{2}\right\rangle+\left\langle x, w, w_{1} w_{2}\right\rangle-\left\langle x, w, w_{1}\right\rangle
\end{aligned}
$$


while

$$
\begin{aligned}
s \circ \partial\left\langle x, w, w_{1}, w_{2}\right\rangle= & x w s\left\langle w_{1}, w_{2}\right\rangle-s\left\langle x w w_{1}, w_{2}\right\rangle+s\left\langle x w, w_{1} w_{2}\right\rangle-s\left\langle x w, w_{1}\right\rangle \\
= & x w s\left\langle w_{1}, w_{2}\right\rangle-x s\left\langle w w_{1}, w_{2}\right\rangle+\left\langle x, w w_{1}, w_{2}\right\rangle \\
& +x s\left\langle w, w_{1} w_{2}\right\rangle-\left\langle x, w, w_{1} w_{2}\right\rangle-x s\left\langle w, w_{1}\right\rangle+\left\langle x, w, w_{1}\right\rangle \\
= & x s \circ \partial\left\langle w, w_{1}, w_{2}\right\rangle+\left\langle x, w w_{1}, w_{2}\right\rangle-\left\langle x, w, w_{1} w_{2}\right\rangle+\left\langle x, w, w_{1}\right\rangle
\end{aligned}
$$

Then,

$$
\begin{aligned}
(\partial \circ s+s \circ \partial)\left\langle x w, w_{1}, w_{2}\right\rangle & =x(\partial \circ s+s \circ \partial)\left\langle w, w_{1}, w_{2}\right\rangle-x\left\langle w, w_{1}, w_{2}\right\rangle \\
& +\left\langle x w, w_{1}, w_{2}\right\rangle-\left\langle x, w w_{1}, w_{2}\right\rangle+\left\langle x, w, w_{1} w_{2}\right\rangle \\
& -\left\langle x, w, w_{1}\right\rangle+\left\langle x, w w_{1}, w_{2}\right\rangle-\left\langle x, w, w_{1} w_{2}\right\rangle+\left\langle x, w, w_{1}\right\rangle
\end{aligned}
$$

Using the inductive assumption, the first two terms cancel and all the rest cancel in pairs, except for $\left\langle x w, w_{1}, w_{2}\right\rangle$, which shows that $s \circ \partial+\partial \circ s=1$ in this case. The second case, that $w_{0}$ begins with the inverse of a letter is similar.

This completes the proof of the homotopy equivalence for group cohomology. For associative algebras the argument is quite similar. For Lie algebras and, especially for commutative algebras, it is a good deal more complicated because it is less obvious that the cochain complex of a free algebras splits. In fact, for Harrison's cochain complex, it splits only in characteristic 0 and the equivalence fails in finite characteristic. As a result, the cohomology that results from the cotriple resolution has been seen as primary.

\section{Acyclic models now}

Besides the acyclic models theorem quoted above, there was a weaker form due to Michel André [André, 1967, 1974] in which the conclusion was the weaker homology isomorphism and one could not infer naturality, at least as it was stated and proved. In the process of trying to settle the naturality question I discovered an acyclic models theorem that included both the version above and André's as special cases, along with at least one other interesting version. I outline the definitions and theorems. For proofs, I refer to my recent book [Barr, 2002].

In this definition, $\mathscr{C}=\mathrm{CC}(\mathscr{A})$ is the category of chain complexes of an abelian category $\mathscr{A}$.

11.1 Acyclic classes. A class $\Gamma$ of objects of $\mathscr{C}$ will be called an acyclic class provided:

AC-1. The 0 complex is in $\Gamma$.

AC-2. The complex $C_{\bullet}$ belongs to $\Gamma$ if and only if $S C_{\bullet}$ does.

AC-3. If the complexes $K_{\bullet}$ and $L_{\bullet}$ are homotopic and $K_{\bullet} \in \Gamma$, then $L_{\bullet} \in \Gamma$.

$\mathrm{AC}-4$. Every complex in $\Gamma$ is acyclic.

AC-5. If $K_{\bullet \bullet}$ is a double complex, all of whose rows are in $\Gamma$, then the total complex of $C$ • belongs to $\Gamma$.

Given an acyclic class $\Gamma$, let $\Sigma$ denote the class of arrows $f$ whose mapping cone is in $\Gamma$. It can be shown that this class lies between the class of homotopy equivalences and that of homology equivalences. 
Suppose that $G: \mathscr{X} \longrightarrow \mathscr{X}$ is an endofunctor and that $\epsilon: G \longrightarrow$ Id is a natural transformation. If $F: \mathscr{X} \longrightarrow \mathscr{A}$ is a functor, we define an augmented chain complex functor we will denote $F G^{\bullet+1} \longrightarrow F$ as the functor that has $F G^{n+1}$ in degree $n$, for $n \geq-1$. Let $\partial^{i}=F G^{i} \epsilon G^{n-i}: F G^{n+1} \longrightarrow F G^{n}$. Then the boundary operator is $\partial=\sum_{i=0}^{n}(-1)^{i} \partial^{i}$. If, as usually happens in practice, $G$ and $\epsilon$ are $2 / 3$ of a cotriple, then this chain complex is the chain complex associated to a simplicial set built using the comultiplication $\delta$ to define the degeneracies. Next suppose that $K_{\bullet} \longrightarrow K_{-1}$ is an augmented chain complex functor. Then there is a double chain complex functor that has in bidegree $(n, m)$ the term $K_{n} G^{m+1}$. This will actually commute since

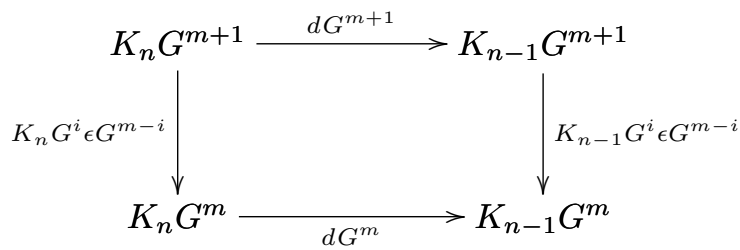

commutes by naturality for $0 \leq i \leq m$ hence so does

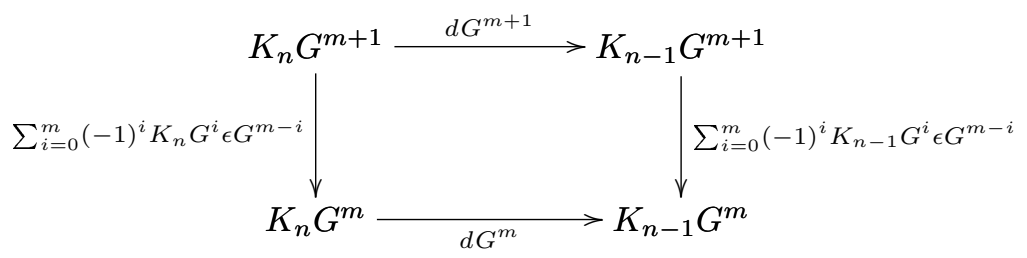

However, the usual trick of negating every second column produces an anticommuting double complex.

This is augmented in both directions, once, using $\epsilon$, over the single complex $K$. and second, using the augmentation of $K_{\bullet}$, over the complex $K_{-1} G^{\bullet+1}$. We say that $K_{\bullet}$ is $\epsilon$-presentable with respect to $\Gamma$ if for each $n \geq 0$, the augmented chain complex $K_{n} G^{\bullet+1} \longrightarrow K_{n} \longrightarrow 0$ belongs to $\Gamma$. We say that $K_{\bullet}$ is $G$-acyclic with respect to $\Gamma$ if the augmented complex $K_{\bullet} G \longrightarrow K_{-1} G \longrightarrow 0$ belongs to $\Gamma$.

It could be notes that an augmented chain complex $C_{\bullet} \longrightarrow C_{-1} \longrightarrow 0$ is the desuspension of the mapping cone of the map of chain complexes $C_{\bullet} \longrightarrow C_{-1}$ in which the latter is considered as a chain complex with $C_{-1}$ in degree 0 and 0 in all other degrees. Thus an equivalent formulation of the two definitions above is that $K_{\bullet}$ is $\epsilon$-presentable with respect to $\Gamma$ if for each $n \geq 0$, the chain map $K_{n} G^{\bullet+1}$ $\longrightarrow K_{n}$ belongs to $\Sigma$ and that $K_{\bullet}$ is $G$-acyclic with respect to $\Gamma$ if the chain map $K_{\bullet} G \longrightarrow K_{-1} G \longrightarrow 0$ belongs to $\Sigma$.

Theorem 4 Let $\Gamma$ be an acyclic class and $\Sigma$ be the associated class of arrows. Suppose $\alpha: K_{\bullet} \longrightarrow K_{-1}$ and $\beta: L_{\bullet} \longrightarrow L_{-1}$ are augmented chain complex functors. Suppose $G$ is an endofunctor on $\mathscr{X}$ and $\epsilon: G \longrightarrow \operatorname{Id}$ a natural transformation for which $K_{\bullet}$ is $\epsilon$-presentable and $L_{\bullet} \longrightarrow L_{-1} \longrightarrow 0$ is $G$-acyclic, both with respect to $\Gamma$. Then given any natural transformation $f_{-1}: K_{-1} \longrightarrow L_{-1}$ there is, in $\Sigma^{-1} \mathscr{C}$, a unique arrow $f_{\bullet}: K_{\bullet} \longrightarrow L_{\bullet}$ that extends $f_{-1}$. 
Although we give no proof, here is the "magic diagram" from which it all follows:

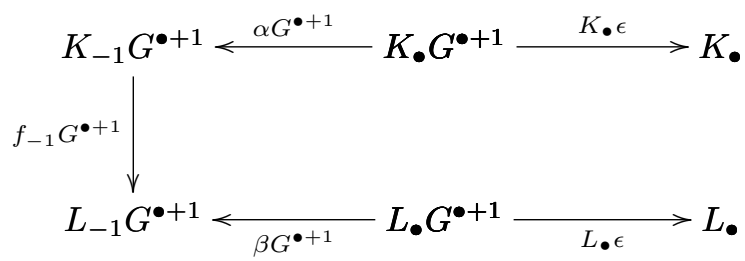

Note that each of the two hypotheses implies that one of the "wrong way" arrows belongs to $\Gamma$. When these are inverted, you get a map $K_{\bullet} \longrightarrow L_{\bullet}$.

11.2 Examples. We mention three examples of acyclic classes.

Let $\Gamma$ be the class of contractible complexes. In that case, $\Sigma$ is the class of homotopy equivalences. It is trivial to show that when the rows and columns of a double complex are contractible so is the total complex. It is a little more surprising - but still true - that if the rows or the columns are contractible, so is the total complex. The only other point to make is that in this case, the functor $\mathscr{C}$ $\longrightarrow \Sigma^{-1} \mathscr{C}$ is surjective on arrows, so that you actually get a homotopy equivalence as conclusion.

Let $\Gamma$ be the class of acyclic complexes. In that case, $\Sigma$ is the class of chain maps that induce an isomorphism on homology (called homology isomorphisms). One can use a trivial spectral sequence argument to show that if all the rows or all the columns of a double complex are acyclic, then so is the total complex, but it is not hard to give a direct argument using a filtration. Since the functor $\mathscr{C}$ $\longrightarrow \Sigma^{-1} \mathscr{C}$ is not surjective on arrows, the arrows you get are not induced by arrows between the chain complexes, but they are natural.

Let $\Gamma$ be the class of chain complexes that are, at each object of $\mathscr{X}$, contractible, but not naturally so. In that case, $\Sigma$ consists of arrows that induce, at each $X$, homotopy equivalences. This means that the arrows are natural, but the homotopy inverse and the homotopies involved are not necessarily natural. This situation arises quite naturally in topology. For example, on the category of $C^{\infty}$ manifolds, the inclusion of the $C^{\infty}$ chains into all the chains can be shown to induce a homotopy equivalence on any space, but there is no obviously natural way of doing this. This example answered a question raised by Rob Milson who was working with these homology groups.

\section{References}

M. André (1967), Méthode simpliciale en algèbre homologique et algèbre commutative. Lecture Notes in Mathematics 43, Springer-Verlag, Berlin, Heidelberg, New York.

M. André (1974), Homologie des algèbres commutatives. Springer-Verlag, Berlin, Heidelberg, New York.

H. Appelgate (1965), Categories with models. Dissertation, Columbia University.

R. Baer (1934), Erweiterungen von Gruppen und ihren Isomorphismem. Math. Zeit. 38, 375-416.

M. Barr (1962), On the cohomology of commutative algebras. Dissertation, University of Pennsylvania.

M. Barr (1965a), A cohomology theory for commutative algebras, I. Proc. Amer. Math. Soc. 16, 1379-1384.

M. Barr (1965b), A cohomology theory for commutative algebras, II. Proc. Amer. Math. Soc. 16, 1385-1391. 
M. Barr (1966), Cohomology in tensored categories. Proceedings of the Conference on Categorical Algebra, Springer-Verlag, Berlin, Heidelberg, New York, 344-354.

M. Barr (1967), Shukla cohomology and triples. J. Algebra 5, 222-231.

M. Barr (1968a), A note on commutative algebra cohomology. Bull. Amer. Math. Soc. 74, 310313.

M. Barr (1968b), Harrison homology, Hochschild homology and triples. J. Algebra, 8, 314-323.

M. Barr (1969), Cohomology and obstructions: commutative algebras. In B. Eckmann, ed. Seminar on Triples and Categorical Homology Theory, Lecture Notes in Mathematics 80, 357-375, Springer-Verlag, Berlin, Heidelberg, New York.

M. Barr (1996a), Cartan-Eilenberg cohomology and triples. J. Pure Applied Algebra, 112, 219238.

M. Barr (1996b), Acyclic models. Canadian J. Math., 48, 258-273.

M. Barr (2002), Acyclic Models. Centre de Recherche Mathématiques.

M. Barr (forthcoming), Resolutions and derived functors. ftp.math.mcgill.ca/pub/barr/ derfun. $\{$ ps,pdf $\}$.

M. Barr and J. Beck (1966), Acyclic models and triples. Proceedings of the Conference on Categorical Algebra, Springer, 336-343.

M. Barr and J. Beck (1969), Homology and standard constructions. In M. Tierney and A. Heller, eds., Seminar on Triples and Categorical Homology Theory, Lecture Notes in Mathematics 80, Springer-Verlag, Berlin, Heidelberg, New York, 245-535.

M. Barr and M.-A. Knus (1971), Extensions of Derivations. Proc. Amer. Math. Soc. 28, 313-314. M. Barr, and G. S. Rinehart (1966), Cohomology as the derived functor of derivations. Trans. Amer. Math. Soc., 122, 416-426.

D. Buchsbaum (1956), Exact categories. Appendix to [Cartan and Eilenberg, 1956].

H. Cartan and S. Eilenberg (1956), Homological Algebra. Princeton University Press.

C. Chevalley and S. Eilenberg (1948), Cohomology theory of Lie groups and Lie algebras. Trans. Amer. Math. Soc., 63, 85-124.

S. Eilenberg and S. Mac Lane (1941), Infinite cycles and homologies. Proc. Nat. Acad. Sci. U. S. A., 27, 535-539.

S. Eilenberg and S. Mac Lane (1942a), Natural isomorphisms in group theory. Proc. Nat. Acad. Sci. U. S. A., 28, 537-543.

S. Eilenberg and S. Mac Lane (1942b), Group extensions and homology. Ann. of Math., 43, 757-831.

S. Eilenberg and S. Mac Lane (1943), Relations between homology and homotopy groups. Proc. Nat. Acad. Sci. U. S. A., 29, 155-158.

S. Eilenberg and S. Mac Lane (1945a), General theory of natural equivalences. Trans. Amer. Math. Soc., 58, 231-294.

S. Eilenberg and S. Mac Lane (1945b), Relations between homology and homotopy groups of spaces. Ann. of Math., 46, 480-509.

S. Eilenberg and S. Mac Lane (1947a), Cohomology theory in abstract groups, I. Ann. of Math., 48, 51-78.

S. Eilenberg and S. Mac Lane (1947b), Cohomology theory in abstract groups, II. Group extensions with a non-Abelian kernel. Ann. of Math., 48, 326-341.

S. Eilenberg and S. Mac Lane (1951), Homology theories for multiplicative systems. Trans. Amer. Math. Soc., 71, 294-330.

S. Eilenberg and S. Mac Lane (1953), Acyclic models. Amer. J. Math., 75, 189-199.

S. Eilenberg and J. C. Moore (1965a), Foundations of Relative Homological Algebra, Memoirs Amer. Math. Soc. 55.

S. Eilenberg and J. C. Moore (1965b), Adjoint functors and triples. Illinois J. Math., 9, 381-398. Murray Gerstenhaber and S. D. Schack (1987), A Hodge-type decomposition for commutative algebra cohomology. J. Pure Appl. Algebra 48, 229-247. 
R. Godement (1958), Topologie algébrique et théorie des faisceaux. Actualités Sci. Ind. No. 1252. Publ. Math. Univ. Strasbourg, 13, Hermann, Paris.

A. Grothendieck (1957), Sur quelques points d'algèbre homologique. Tohôku Math. Journal 2, $119-221$.

M. Hall (1938), Group rings and extensions, I. Ann. of Math. 39, 220-234.

D. K. Harrison (1962), Commutative algebras and cohomology. Trans. Amer. Math. Soc., 104, 191-204.

G. Hochschild (1945), On the cohomology groups of an associative algebra. Ann. of Math. (2), 46, 58-67.

G. Hochschild (1946), On the cohomology theory for associative algebras. Ann. of Math. (2), 47, $568-579$.

H. Hopf $(1942,1943)$, Fundamentalgruppe und zweite Bettische Gruppen. Comment. Math. Helv. 14, 257-309. Nachtrag, 15, 27-32.

H. Hopf (1945), Ueber die Bettischen Gruppen die zu einem beliebigen Gruppe gehören. Comment. Math. Helv. 17, 39-79.

P. Huber (1961), Homotopy theory in general categories. Math. Ann., 144, 361-385.

H. Kleisli (1964), Comparaison de la résolution simpliciale à la bar-résolution. In Catégories Non-Abéliennes, L'Université de Montréal, 85-99.

H. Kleisli (1965), Every standard construction is induced by a pair of adjoint functors. Proc. Amer. Math. Soc., 16, 544-546.

S. Mac Lane (1950), Duality for groups. Bull. Amer. Math. Soc. 56, 485-516.

S. Mac Lane (1958), Extensions and obstructions for rings. Ill. J. Math. 2, 316-345.

S. Mac Lane (1963), Homology. Springer-Verlag, Berlin, Heidelberg, New York.

U. Shukla (1961), Cohomologie des algèbres associatives. Ann. Sci. École Norm. Sup. (3), 78, 163-209.

A. M. Turing (1938), The extensions of a group. Compositio Math. 5, 357-367.

H. Zassenhaus (1937), Lehrbuch der Gruppentheorie. Hamburg Math. Einzelschriften 21, Leipzig. 